\title{
ELECTRON CLOUD STUDIES AND ANALYSES AT SPS FOR LHC-TYPE BEAMS
}

\author{
J.M. Jimenez, G. Arduini, V. Baglin, P. Collier, G. Ferioli, B. Henrist, N. Hilleret, L. Jensen, \\ B. Jenninger, J.M. Laurent, A. Rossi, K. Weiss, F. Zimmermann \\ AT / AB Collaboration, CERN, Geneva, Switzerland
}

\begin{abstract}
A summary of the main results obtained so far from the electron cloud studies using strip detectors, pick-ups, COLDEX and a $100 \mathrm{MHz}$ coaxial resonator will be presented. The spatial and energy distributions of the electrons in the cloud measured by the strip detectors will be detailed and compared to the results obtained with a conventional retarding field detector. The evidence of the scrubbing effect and of the NEG coatings as remedies to reduce the electron cloud activity will also be shown.

In a second part, the improved hardware of the experiments will be presented together with the program of measurements foreseen for the 2003 SPS run.
\end{abstract}

\section{SET UP \& MAIN RESULTS}

\subsection{Electron Cloud thresholds, build up}

\subsubsection{Set up description}

Since 1998, the electron cloud activity is being studied in the SPS with LHC-type beams ${ }^{1}$ using the pressure gauges and shielded pick-ups [1][2][3]. During the shutdown of 2001-02, three different versions of stripdetectors have been installed to study independently or simultaneously the spatial distribution and the energy distributions of the electrons in the cloud [4].

\subsubsection{Electron Cloud thresholds, build up}

The electron cloud multipacting is driven mainly by the beam parameters and by the secondary electron yield $\left(\delta_{\max }\right.$, Energy of the maximum of the secondary electron yield) of the wall surface.

Immediately after the air venting during the 2001/02 shutdown, the threshold of the electron cloud, measured with a single batch-injection, was $3.0 \times 10^{10} \mathrm{p} /$ bunch in the dipole field regions and $5.5 \times 10^{10} \mathrm{p} /$ bunch for the fieldfree. In a dipole field, the appearance threshold of the two lateral strips was $5.5 \times 10^{10} \mathrm{p} /$ bunch and at $1.3 \times 10^{11}$ $\mathrm{p} /$ bunch, a $3^{\text {rd }}$ central strip appeared as predicted by the simulations [5][6]. The observations showed that the central strip tends to disappear after a few hours of scrubbing and an attempt of explanation will be given in $\S 1.4$. Fig.1 shows the electron cloud build up in a dipole field with 4 batches injected.

The electron cloud build up is strongly dependent on the beam potential (bunch intensity and length) which

\footnotetext{
1 1-4 batches of 72 bunches injected from the PS machine, 25 ns bunch spacing, $225 \mathrm{~ns}$ batch spacing, $1.3 \times 10^{11}$ protons/bunch $\left(1.7 \times 10^{11}\right.$ ultimate) and $4 \mathrm{~ns}$ bunch length $(4 \sigma)$ at injection energy $(26 \mathrm{GeV})$.
}

determines the kick to the electrons. As expected, the measurements showed an increase of the electron cloud intensity by a factor of 7 to 10 when the bunch intensity is doubled from $5.0 \times 10^{10}$ to $1.1 \times 10^{11} \mathrm{p} /$ bunch.

Similarly, a decrease of the bunch length by $30 \%$ doubles the electron cloud intensity (Fig.2) and a $3^{\text {rd }}$ central strip appears already at $1.1 \times 10^{11} \mathrm{p} /$ bunch instead of $1.3 \times 10^{11} \mathrm{p} / \mathrm{bunch}$ for the nominal bunch length (4 ns). Conversely, if the bunch length is increased by $30 \%$, the electron cloud disappeared (Fig.2).

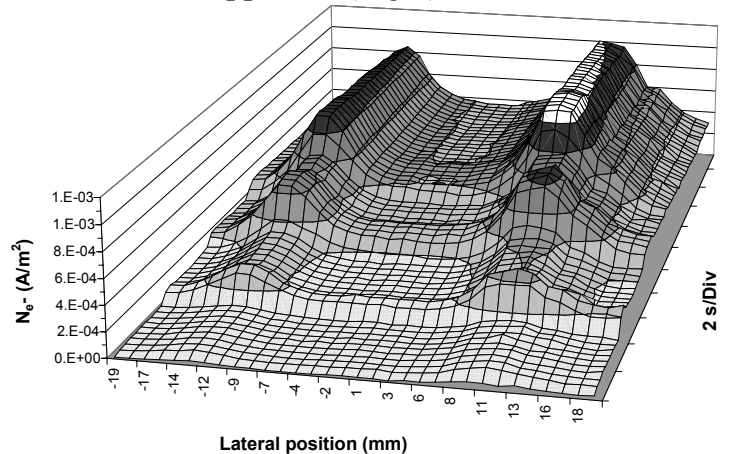

Fig.1: Electron cloud signal measured using the stripdetector in a dipole field with 4 batches injected.
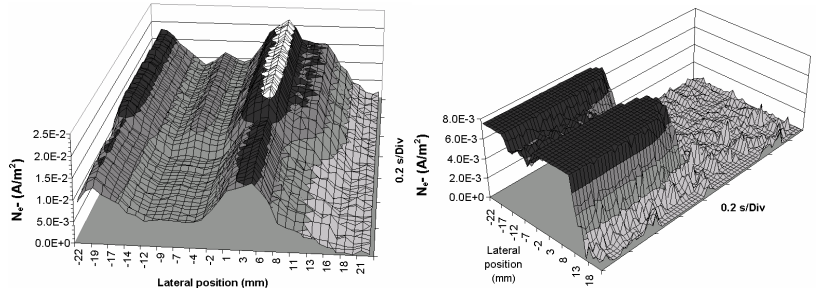

Fig.2: The electron cloud is enhanced by a bunch length decrease (left,-30\%), and disappears with an increase of the bunch length (right, $+30 \%$ ).

\subsubsection{Multi-batch - Surviving electrons}

To optimise the injection of the LHC, 3 or 4 batches will be accumulated in the SPS before being injected into the LHC. The measurements made using the pick-ups confirmed that the electron cloud build up during the passage of the $2^{\text {nd }}$, the $3^{\text {rd }}$ and $4^{\text {th }}$ batch is enhanced by the passage of the preceding batches (Fig.3). If the lifetime of the electrons is higher than the $225 \mathrm{~ns}$ batch spacing, the surviving electrons created during the previous batch passage will enhance the build up during the passage of the following batches. But after the $4^{\text {th }}$ batch passage and due to the revolution time in the SPS, i.e. $23 \mu \mathrm{s}$, the $1^{\text {st }}$ batch will pass once again after $14.6 \mu$ s. This delay 
appears as long enough to loose all the surviving electrons.

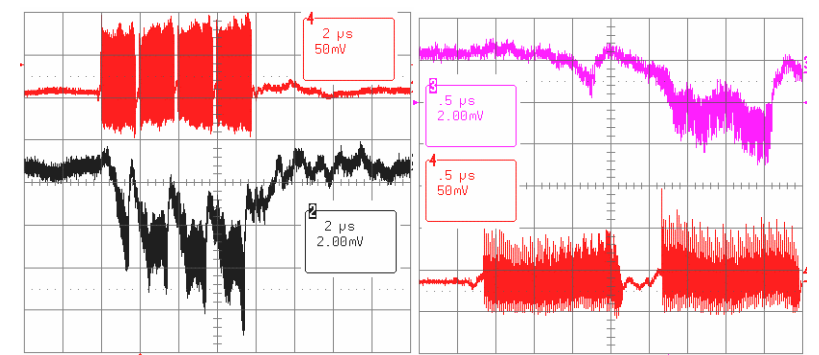

Fig.3: Pick-up signal: build up during the passage of the $2^{\text {nd }}, 3^{\text {rd }}$ and $4^{\text {th }}$ batch enhanced by the passage of the preceding batches (left). A $550 \mathrm{~ns}$ batch spacing did not decouple the build up of two successive batches (right).

\subsubsection{Scrubbing Effect}

Two scrubbing periods, 15 days of beam in total, gave evidence of a "scrubbing effect". All around the SPS, both in the field-free and dipole field regions, the pressures decreased by $10^{3}$ in 4 days, $10^{4}$ in 10 days (Fig.4).

Meanwhile, the threshold in the dipole field increased from $3.0 \times 10^{10}$ to $1.0 \times 10^{11} \mathrm{p} / \mathrm{bunch}$ and from $5.0 \times 10^{10}$ to more than $1.3 \times 10^{11} \mathrm{p} / \mathrm{bunch}$ in the field-free regions. No signal could be detected in the field-free regions after 10 days of LHC-type beams with a 4 batches injected.

Similarly, the evolution of the electron cloud activity measured by the strip-detectors throughout the cleaning process showed a decrease of the electron flux by a factor $10^{2}$ in 10 days in a field-free region.

Several parameters measured (pressures, electron cloud intensity, heat load measured by the calorimeters [7][8]) showed that the beam-induced multipacting and thus the scrubbing stopped after 4 days in the field-free regions which is consistent with the in situ measurement of the secondary electron yield (SEY), which remained constant after 4 days; the $\delta_{\max }$ was between 1.5 and 1.6. After short periods without LHC-type beams, the SEY drifted up i.e. from 1.5 to 1.7 in two weeks time. However, the initial value was recovered after 4 hours with nominal intensity LHC-type beams with at least 3 batch-injections.

\subsubsection{Effect of the ramp in energy}

At the end of the scrubbing period, a small ramp in energy was introduced after the $4^{\text {th }}$ batch-injection; the proton energy was increased up to $55 \mathrm{GeV}$ to check whether or not the scrubbing run should be made at injection energy (costs of operation) or if unexpected enhancement effects will imply going to higher energies.

The effect of this small ramp was bigger than expected since it induced a small displacement of the orbit (Fig.5) producing pressure rises all around the SPS machine and an enhancement of the electron cloud intensity measured by the strip-detectors.

A squeezing of the bunch during the ramp, which implies an increase of the beam potential, and therefore an increase of the electron energies could easily explain the increase of the electron cloud activity. The pressure rises can be explained by the orbit displacement, which involved new "less scrubbed" surfaces. This hypothesis is consistent with the measurements made by introducing a small orbit displacement between 3 to $4 \mathrm{~mm}$ with respect to the nominal orbit, which produced an increase of the pressures $\Delta \mathrm{P} / \mathrm{P}$ by 3 to 5 depending on the chamber shape and position in the SPS ring. The pressure rises clearly indicate that the electrons are bombarding non-scrubbed surfaces; Consistently, the electron cloud activity measured by the strip-detectors increased by more than a factor 2 .

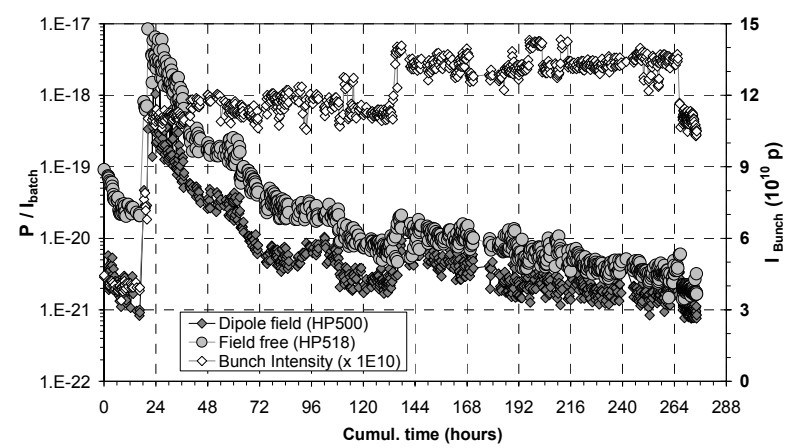

Fig.4: Pressure decreases both in dipole and field-free regions with the LHC-type beam exposure. The pressures are normalised to the batch intensity $\left(P / I_{\text {bunch }} \mathrm{x} 72 \mathrm{x} N_{\text {bacthes }}\right)$.

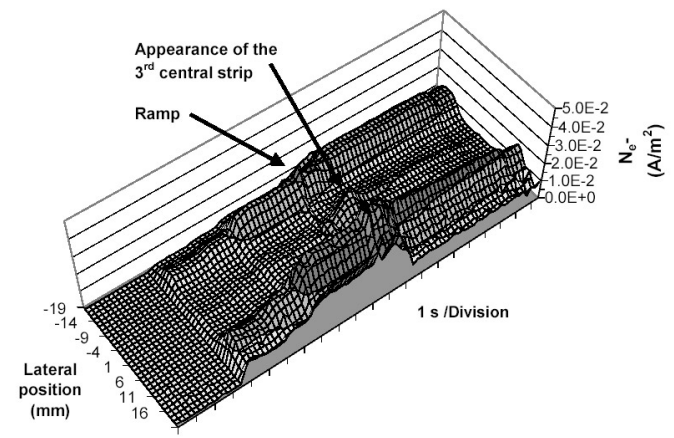

Fig.5: Small orbit displacement seen by the stripdetectors due to the ramp to $55 \mathrm{GeV}$.

\subsection{Vacuum behaviour of cold surfaces COLDEX}

The COLDEX experiments, which was previously installed in the EPA ring (PS Complex) to measure the photon stimulated desorption, has been installed in a field-free region of the SPS (HP417) in May 2002. The aim of this experiment was to study the dynamic pressure evolutions in presence of LHC-type beams at cold (5-20 $\mathrm{K})$ with a beam screen design close to the LHC one.

The COLDEX beam screen was made out of OFE copper with a length of $2.2 \mathrm{~m}$ and with an elliptic shape $(\mathrm{H}=84 \mathrm{~mm}, \mathrm{~V}=66 \mathrm{~mm})$. A $1 \%$ of the total surface is composed of holes, which allow the transfer of gases from the beam screen to the cold bore. The cold bore is operated between 3 to $5 \mathrm{~K}$ while the beam screen could be operated from 5 to $100 \mathrm{~K}$.

UHV pressure gauges, residual gas analyser and gas injection (Fig.6) allow following the partial and total 
pressures. The heat load is measured on the cryogenic cooling circuit.

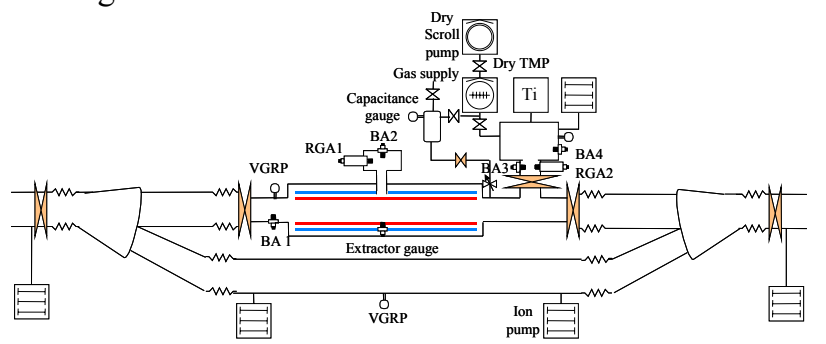

Fig.6: Schematic view of the COLDEX experiment

The pressure in COLDEX also suggests a scrubbing effect; the pressure decreased by $10^{2}$ after 4 days (Fig.7) reaching the equivalent of 100 hours beam lifetime in the LHC. During all the evolution, the beam screen was kept around $8 \mathrm{~K}$ and the cold bore at $5 \mathrm{~K}$. The increase during a few hours of the beam screen temperature to $120 \mathrm{~K}$ did not change the picture; the same result was obtained after the injection of one monolayer of hydrogen.

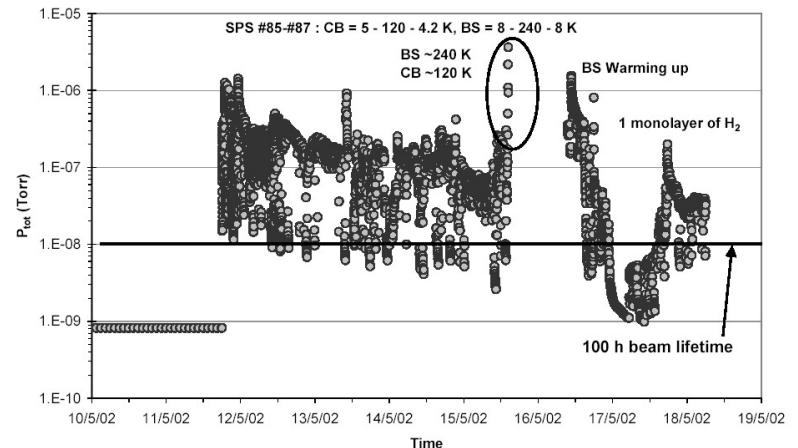

Fig.7: Pressure evolution in the COLDEX during the $1^{\text {st }}$ scrubbing run.

The preliminary results obtained on the heat loads measured by COLDEX (95\% duty cycle, $1.1 \times 10^{11}$ $\mathrm{p} /$ bunch) still need confirmation since the heat load measured is larger than the available cooling power in the LHC. The simulations made for a similar geometry and assuming a SEY of 1.4 gave similar results [6].

\subsection{NEG: A remedy to the Electron Cloud}

The use of NEG pumping coatings (TiZrV) [9] has been approved as the vacuum baseline for the vacuum chambers of the LHC room temperature long strait sections. It was expected that the low secondary electron yield of the NEG surface after activation $(\delta=1.1)[10]$ and even if saturated $(\delta=1.2$ with a $\mathrm{CO}$ saturation) [11], should decrease the electron cloud.

To validate the NEG behaviour, a test bench was installed in the SPS with a symmetrical layout allowing a direct comparison between NEG coated chambers and stainless steel chambers with identical shapes [12].

Fig. 8 shows the difference in behaviour between a nonactivated NEG and an activated and saturated NEG. With the non-activated NEG, an electron current is measured by the shielded pick-ups and the NEG coated chamber behaves like the reference stainless steel chamber. After activation and even if fully saturated (water coming from the non-baked upstream and downstream vacuum chambers), the results confirmed that no electron cloud signal is visible at nominal bunch intensity with 4 batches injected. In presence of beam, the predominant gases are, as expected: $\mathrm{H}_{2}, \mathrm{CO}, \mathrm{CO}_{2}$ and $\mathrm{CH}_{4}$.
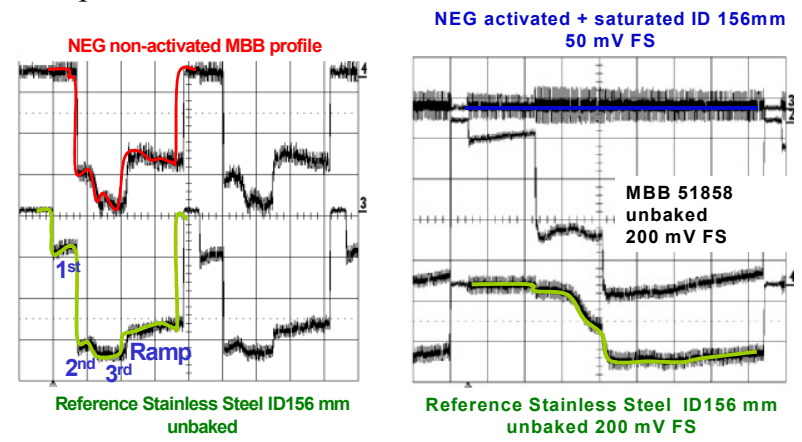

Fig.8: Electron cloud signals measured using pick-ups on a reference stainless steel chamber as compared to a nonactivated NEG coated chamber (left) and to an activated and saturated NEG coated chamber (right).

\subsection{Spatial and Energy distributions}

The spatial distribution of the electrons in the cloud has been confirmed using a 36-channels strip-detector, which allowed twice the resolution, obtained in 2001 and recent simulations fit fairly well the measurements [13]. At $1.1 \times 10^{11}$ and $1.3 \times 10^{11} \mathrm{p} / \mathrm{bunch}$, the two vertical lateral strips of the cloud will stand on top of the beam screen pumping slots $(9$ and $11.5 \mathrm{~mm}$ respectively from the center) inducing additional heat load to the cold bore. The decision to insert a pumping slot shielding to intercept the electrons passing through the slots was accepted and is now in the LHC baseline [14].

The energy distribution of the electrons in the cloud was measured using the strip-detectors in both the dipole field and field-free conditions and using a conventional retarding field detector in a field-free region. Due to hardware limitations, the electrons with energies below 20 $\mathrm{eV}$ could not be measured. However, the impact of this limitation is small both on the heat load budget and on the pressure rises induced by the electron stimulated desorption (ESD) mechanism since for both processes, the expected contribution of the low energy electrons $(<20 \mathrm{eV})$ is small $(<20 \%)$.

In the dipole field where most of the electrons are trapped in two vertical lateral strips by the magnetic field, the energy distribution showed a peak between 180 and $200 \mathrm{eV}$ to be compared with the $80 \mathrm{eV}$ measured in the field-free regions.

The simultaneous energy and spatial distributions studies showed that most of the high-energy electrons i.e. above $200 \mathrm{eV}$, are located in the central strip (Fig.9). The two lateral strips have electrons with energies below 180 $\mathrm{eV}$. This last observation could explain why the central strip tends to disappear after several hours of LHC-type beam. In fact, the decrease of the secondary electron yield is enhanced by the amount of primary electrons. After a 
given dose, the amount of electrons produced, i.e. $\delta_{\mathrm{SEY}}$ above the multipacting threshold is reached earlier for the electrons with energies above $800 \mathrm{eV}$ thus causing the extinction of the cloud in the central area.

\subsection{Coaxial resonator $100 \mathrm{MHz}$}

Multipacting measurements were also performed in the Laboratory without beam, using a $100 \mathrm{MHz}$ coaxial resonator. The external conductor of the resonant cavity (Fig.10) is a $1500 \mathrm{~mm}$ in length (DN100) stainless steel vacuum chamber. A lateral cut-off tube (DN35) allows connecting an electron pick-up in the central part of the resonator, where the electric field is maximized in the $1 / 2$ mode [15]. A stainless steel grid, perforated by $2 \mathrm{~mm}$ holes, closes this port in order to maintain the chamber wall continuity and to shield the pickup from the RF signal. This grid is treated in the same way as the chamber.

The onset of multipactor is detected by the observation of several changes in the cavity behaviour, which happen when the input power reaches the multipactor threshold. Those following changes constitute the cavity multipactor signature: collection of electrons by the pick-up, a simultaneous increase of the vacuum pressure, a saturation of the output signal, an increase of the reflected signal, a detuning of the cavity and the generation of harmonics.

If baked below the activation threshold $\left(150^{\circ} \mathrm{C}\right)$, the multipactor threshold of the NEG coated chamber is similar to the one of an unbaked stainless steel. After the activation of the NEG coating, the multipacting threshold increased and the amount of electrons collected by the pick-up decreased. Fig.11 shows how the output voltage trend is modified above a given input power threshold due to the adsorption of a fraction of the input power by the multipacting electrons. When activated, the output signal increases linearly with the input power indicating the absence of electrons. The same measurements were made at liquid nitrogen temperature $(77 \mathrm{~K})$ and with an activated NEG, no electron multipacting was measured (Fig.11). Even intentioned cryosorbtion of gases (CO, $\mathrm{CO} 2$ and air) onto the inner wall of the cavity did not change the picture.

\section{OBJECTIVES FOR 2003}

The vacuum studies at cryogenic temperature will be the priority of the new measurement campaign. The aim is to study the effect of surface coverage on the vacuum stability. The proof of existence of a scrubbing effect at cryogenic temperature is also a major issue since the scrubbing was only confirmed at room temperature. These experiments will be carried out using the COLDEX experiment equipped with a beam screen design closer to the LHC baseline. The higher order modes shielding has been improved to avoid any contribution to the heat load measured by COLDEX using a dedicated thermometry. An RT calorimeter with a similar aperture will allow comparing heat loads at cold and room temperature. The measurements at cryogenic temperature will also rely on the results given by a new cold strip-detector, which is a strip-detector operating between 20 and $30 \mathrm{~K}$ installed in a remotely controlled dipole corrector.

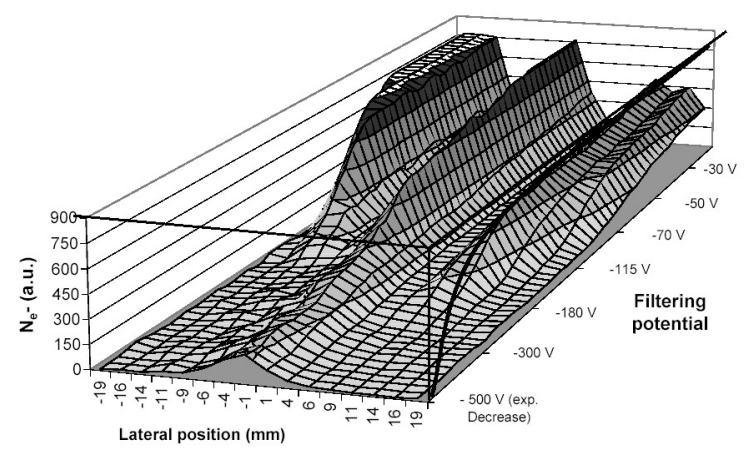

Fig.9: Spatial energy distribution $\left(d^{2} N / d x d E\right)$ of the electrons measured in a single-cycle mode using the retarding field strip-detector.

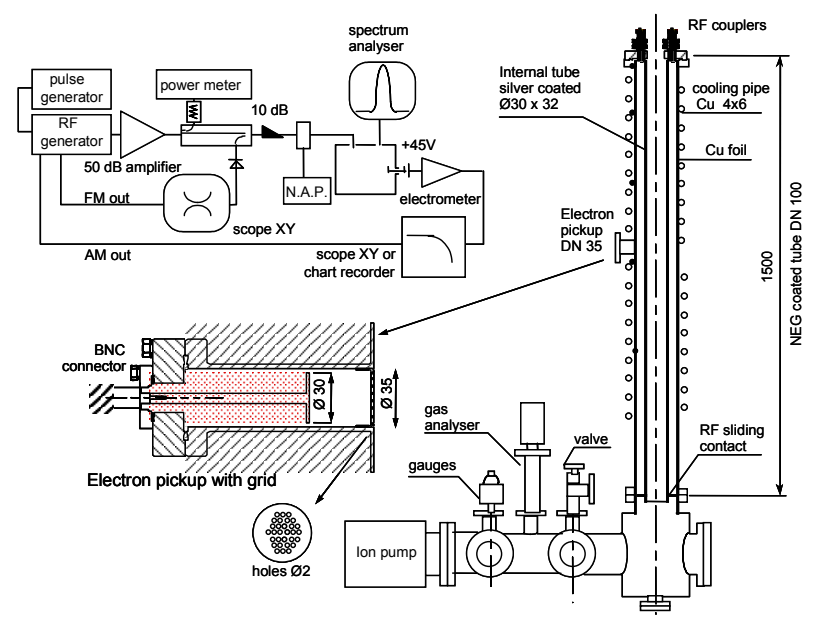

Fig.10: Schematic view of the $100 \mathrm{MHz}$ coaxial resonator

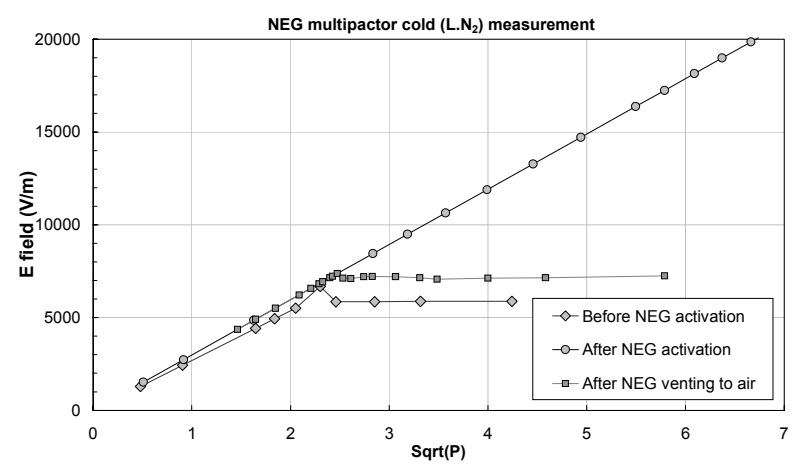

Fig.11: Multipacting measured on a NEG coated chamber at liquid nitrogen temperature.

For the LHC, heat loads will be an issue and the scaling from the SPS results to the LHC at room and cryogenic temperature, in dipole and field-free are essentials. Benchmarking the simulations is also necessary and the dependence of the electron cloud build up on the height of the vacuum chamber will be studied using a variable aperture strip-detector. This strip-detector operating at RT in a remotely controlled dipole corrector has two moving 
plates and allows apertures between 50 and $85 \mathrm{~mm}$ in the vertical plane. The exchangeable plates will allow testing solutions to cure the electron cloud.

A remotely controlled dipole magnet installed on top of the SEY detector will allow studying the evolution of the SEY in a dipole field condition. Since the electron cloud threshold is lower in dipole field conditions, an additional decrease of the SEY is expected.

The NEG coatings used in the LHC LSS appear to be a remedy for the electron cloud. The SPS NEG test bench will be used again to validate the preliminary measurements obtained in 2002. A cycled and saturated NEG will also be studied and compared to a fresh NEG coating.

Finally, the parameters influencing the electron cloud build up will be studied, like the cloud intensity at ultimate bunch intensity, the effect of 6 homogeneously distributed batches in the SPS to verify if the electrons can survive a $3 \mu$ s batch spacing, the effect of the $75 \mathrm{~ns}$ bunch spacing and the energy distributions measured with a new retarding field detector with an increased resolution.

\section{CONCLUSIONS AND DISCUSSIONS}

As being the LHC injector, the scrubbing runs confirmed that after 5 days of scrubbing, the SPS should be able to inject 3 or 4 batches at nominal intensity into the LHC.

However, the electron cloud is still "visible" after about 500 hours of LHC-type beams with 4 batch-injections at nominal intensity but only in the arcs (dipole field regions) both as pressure rises and on the electron signals collected by the strip-detectors. In the field-free regions, i.e. in the long straight sections, the electron cloud activity decreased below the detection level of the stripdetectors $\left(<10^{-9} \mathrm{~A} / \mathrm{m}\right)$. This observation is not in contradiction with the scrubbing efficiency since most of the LHC beam time used for the scrubbing was with 1 or 2 batches injected with bunch intensities close to the electron cloud threshold after 4 days of scrubbing. The scrubbing efficiency depends on the amount and energy of the impinging electrons (bunch intensity, bunch length, number of batches). Similarly to RF and HV devices, the scrubbing efficiency is limited by the parameters used during the scrubbing. The scrubbing will not be effective if running conditions are more favourable for the electron cloud build up.

The ultimate SEY value reached after 4 days in the field-free regions in the SPS $(\delta=1.5)$ shows the multipacting threshold under these conditions. Analytical calculations [16] made recently fit fairly well to the measurements. The upward drift of the SEY $(\delta)$ observed if the SPS is not operated with LHC-type beams is not an issue since measurements confirmed that the SEY recovered its initial value after 4 hours of operation with LHC-type beams above the electron cloud threshold. The detrimental effect of the ramp in energy (up to $450 \mathrm{GeV}$ ) on the electron cloud build up as a consequence of the bunch length shortening and of the beam orbit displacement during the ramp, need to be confirmed and quantified.

For the LHC, the major concern is the excessive heat load due to the electron cloud. Data have been collected and are being compared with the predictions from simulations. However, the LHC situation is expected to be more complicated since most of the machine is at a cryogenic temperature and therefore the gases cryosorbed on the inner surfaces of the vacuum chambers could modify the present picture obtained at room temperature. The scrubbing efficiency is not yet confirmed on cold surfaces on which the energy of the impinging electrons may be decreased by the cryosorbed gases.

The different filling schemes in the two machines also complicate the extrapolation from the SPS situation to the LHC. In the SPS and with 4 batches injected, the surviving electrons are thought to be lost in the $14.6 \mu \mathrm{s}$ between the $4^{\text {th }}$ batch passage and a new passage of the $1^{\text {st }}$ batch. In the LHC [17], the situation could be less favourable since the ring will be full of batches and the maximum spacing between batches will not exceed $3 \mu \mathrm{s}$, corresponding to the rise time of the LHC dump kickers. If the electrons from the cloud survive these gaps, the build up may be significantly enhanced.

\section{REFERENCES}

[1] J.M. Jimenez et al., Workshop Chamonix XI (2001)

[2] J.M. Jimenez et al., PAC01, CHICAGO (2001)

[3] J.M. Jimenez et al., LHC Project Report 632(2003)

[4] J.M. Jimenez et al., LHC Project Report (2003)

[5] F. Zimmermann et al., Workshop Chamonix X (2000)

[6] F. Zimmermann and al., Scrubbing days CERN 2002

[7] N. Hilleret and al, Scrubbing days CERN 2002

[8] V. Baglin, Scrubbing days CERN 2002

[9] C. Benvenuti et al., Vacuum 60 (2001) 57-65.

[10] C. Scheuerlein et al., CERN EST/2000-07 (SM).

[11] B. Henrist et al., CERN Vac. Tech. Note 98-08.

[12] A. Rossi, Scrubbing days CERN 2002

[13] F. Zimmermann, Workshop Chamonix XII (2002)

[14] N. Kos, EDMS 362883 (CERN)

[15] U. Iriso Ariz, Vacuum Technical Note 02-12

[16] L. Vos (private comm. to be published, CERN)

[17] O. Brunning, Minutes of the $59^{\text {th }}$ PLC59 (CERN)

\section{ACKNOWLEDGMENTS}

K. Cornelis ${ }^{1}$, D. Manglunki ${ }^{1}$, SPS \& PS Operators ${ }^{1}$, T. Bohl ${ }^{1}$, P. Baudrenghien ${ }^{1}$, W. Hofle ${ }^{1}$, N. Delruelle ${ }^{2}$, O. Drouyer ${ }^{2}$, D. Legrand ${ }^{2}$, O. Pirrotte ${ }^{2}$, J. Arnold ${ }^{2}$, J-C. Billy ${ }^{2}$, I. Collins', R. Gavaggio ${ }^{2}$, G. Mathis ${ }^{2}$, R. Wintzer ${ }^{2}$, J. Hansen ${ }^{2}$, P. Chiggiato ${ }^{3}$, P. Costa-Pinto ${ }^{3}$, C. Grünhagel ${ }^{3}$, J. Ramillon ${ }^{3}$, N. Munda ${ }^{3}$, G. Favre ${ }^{3}$, D. Valero ${ }^{3}$, M. Blanc and peoples from the Main workshop $^{3}$, M. Doets ${ }^{4}$, P. d. Groen ${ }^{4}$, G. vd Heide ${ }^{4}$, B. Kaan ${ }^{4}$, S. Klous ${ }^{4}$, J. Kuyt ${ }^{4}$, Y. Lefèvre ${ }^{4}$, E.V. Leeuwen ${ }^{4}$, O. Postma ${ }^{4}$, F. Schimmel ${ }^{4}$, C. Zegers ${ }^{4} .{ }^{1} \mathrm{AB},{ }^{2} \mathrm{AT}$ and ${ }^{3} \mathrm{EST}$ Divisions, ${ }^{4}$ NIKHEF for COLDEX 